\title{
El desplazamiento de las fuentes de agregación de valor en la cadena de maíz duro ecuatoriana
}

\author{
Jorge Granda-Aguilar ${ }^{1}$ \\ Fecha de recepción: 29 de Octubre 2018 \\ Fecha de aceptación: 7 de Diciembre 2018
}

\section{Resumen}

Este estudio estima el desplazamiento de las fuentes de agregación de valor en Ecuador según la lógica de la "curva agregada de la sonrisa" que recepta los cambios entre los insumos participes-primarios, secundarios, terciarios-. Con base a los costos de los productores de la cadena de maíz amarillo, se determina ese tipo de desplazamientos mediante una estimación de corte transversal cuya inclinación resulta más a tono con la detectada por Baldwin et. al., 2014 en varios países asiáticos entre 1985-1995, y no tanto la que encuentran entre 19952005 cuando se aprecia una tercerización más clara de la producción.

Palabras clave: Fuentes de agregación de valor, tercerización de la producción, cadena productiva del maíz amarillo

\begin{abstract}
This study determines the displacement in the sources of value aggregation in Ecuador according to the logic of the "smile aggregate curve" that receives the induced alterations between the sources and productive inputs. Based on the cost accounts of the different producers involved in the hard yellow corn chain, this Study determines those variations through a cross-sectional estimate. Evidence of important displacements is found according to the shape that Baldwin et. al., detected between 1985-1995 in several Asian countries and not so much those that is subsequently observed between 1995-2005, when emerges the consolidation of a process of outsourcing of production.
\end{abstract}

Keywords: sources of value aggregation, outsourcing of production, yellow corn chain

\section{Introducción}

La creciente tercerización de la producción a nivel de firma y producto que se asocia con entornos vinculados a los sectores más dinámicos de la economía contemporánea como los de investigación y desarrollo, I+D, marketing, diseño de producto, nuevos materiales, entre otros, sería determinante para la creación progresiva de empleos de calidad de alta remuneración; en las actividades laborales asentadas en las fases de fabricación y ensamblado se alojarían a contrapelo empleos de menor calidad y baja remuneración. Estas manifestaciones del mercado laboral están evidenciando cambios profundos en los procesos subyacentes de agregación de valor donde los componentes de alto rendimiento juegan un rol fundamental a un punto tal que incluyen operaciones graduales de deslocalización de los empleos de bajo valor agregado hacia economías de menor complejidad.

La incorporación de un insumo de alto rendimiento, tipo I+D, en el programa ecuatoriano Semillas orientado hacia agro-cadenas estratégicas, al parecer, estaría en la base para la consecución de progresos significativos en los rendimientos de la producción nacional de maíz amarillo duro, tradicionalmente dependiente de importaciones para el abastecimiento de las demandas originadas en las industrias balanceadora y productora de proteína animal. La producción nacional de maíz, según las cifras de la autoridad responsable, se incrementa 16\%, incluso, desde el primer año de ejecución de ese programa lo que unido a los logros en materia de autoabastecimiento -las importaciones se reducen a 2,4\% de la producción nacional en el 2015 cuando ellas representaban 33,6\% en el 2012- en conjunto determinarían los beneficios atribuidos, entre otros, a ese componente de alto rendimiento inmerso en dicho programa (INEC \& MAGAP, 2015).
Este estudio no profundiza en la conexión entre el incremento de la producción y el programa de mejora competitiva, se concentra, en cambio, en las implicaciones subyacentes a la transferencia de paquetes tecnológicos hacia los productores tradicionales examinando las modificaciones generadas en la composición de los insumos productivos y en los patrones inducidos de agregación de valor, a partir de los cuales se verifica si esos cambios se ajustan a la lógica de tercerización que recoge el contenido de la curva agregada de la sonrisa al comparar los inputs utilizados por los productores beneficiarios frente a los del grupo tradicional no-intervenido donde predomina el uso de insumos primarios.

La evidencia encontrada refleja que la orientación de esos desplazamientos, en realidad, se comportan más a tono con la forma que Baldwin et. al., 2014, detectan para varios países asiáticos, incluido Japón, en el periodo 1985-1995, y no tanto para el siguiente, 1995-2005, en donde se constata el afianzamiento de un proceso de tercerización incompleto de la producción, es decir, el equivalente a una curva cuasi-sonrisa.

Este estudio contiene tres apartados, a saber, el primero revisa la fundamentación detrás del cambio e innovación tecnológica, y profundiza las distinciones conceptuales entre la curva de la sonrisa a nivel de firma "firm-level smile curve" y su versión a nivel agregado "economy-wide smile curve" e incluye los pasos metodológicos que sigue la investigación para estimar los desplazamientos de las fuentes de agregación de valor en Ecuador. El siguiente, ofrece un diagnóstico sucinto de la cadena productiva de maíz amarillo duro en el país e inmediatamente presenta las estimaciones y hallazgos encontrados con base a las cuentas productivas y de costos de productores beneficiarios y no beneficiarios del programa Semillas. En el apartado final aparecen las principales conclusiones que se desprenden de esta primera aproximación sobre los desplazamientos de las fuentes de la agregación de valor en Ecuador.

\section{Metodología}

El sustento conceptual y metodológico detrás de la curva agregada de la sonrisa se arraiga en la necesidad de documentar el dinamismo de los procesos I+D conjuntamente con la desvalorización simultánea de las actividades manufactura y ensamblaje, fruto y consecuencia del proceso de tercerización de la producción y la creciente innovación tecnológica contemporáneas.

En general, el cambio e innovación tecnológica constituyen los vectores que determinan la productividad total de los factores, PTF, la que a su vez, resulta crucial para la comprensión del crecimiento económico. Dentro de una perspectiva mucho más comprehensiva, junto al rol de la PTF, su "causa próxima" emerge como su "causa fundamental" los marcos y entornos institucionales (Acemoglu, 2012). En el momento de explicar las diferencias en la renta per cápita entre economías y países, la evidencia respectiva demuestra que ellas se originarían en las diferencias en la PTF, y, lo propio cuando se analiza las diferencias entre las tasas de crecimiento de la renta per cápita en donde más de la mitad se atribuye a las diferencias en el crecimiento de la PTF. Desde esta perspectiva, por tanto, la teoría del crecimiento económico en sí misma resulta, esencialmente, una teoría del cambio tecnológico (Helpman, p. 54, 2007).

La tercerización creciente de la producción a través de la cual los sectores líderes que albergan el cambio e innovación están decisivamente transformando los patrones de agregación de valor. La metáfora curva 
de la sonrisa -the smile curve- alude a este proceso de tercerización progresivo a nivel de firma. Su intensa dinámica no resulta, sin embargo, tan categórica a nivel agregado donde aparece mucho más difusa ya que se interseca y sobrepone con procesos de diverso origen. En el nivel agregado, para ilustrar, procesos claves de tipo up-stream y down-stream se sobreponen y deslizan con mayor facilidad. En efecto, lo que para una firma en particular representa un insumo-servicio tipo up-stream puede constituir para otra su producto final, por lo tanto se vuelve más nebuloso discriminar si el valor subyacente se está desplazado hacia un proceso up-stream o desde uno tipo down-stream.

Una forma de acotar esa brecha, enfatiza mucho más en el origen sectorial del valor agregado generado en la producción exportable de un país, desagregando entre sus fuentes primarias, secundarias y terciarias; de ese modo se recepta razonablemente la dinámica ascendente de la innovación y tercerización de la economía a través de la curva agregada de la sonrisa (Baldwin et. al., 2014). Con seguridad esta nueva representación soslaya algunos aspectos finos del concepto primigenio a nivel de firma, pero para propósitos de diseño de política, el alcance agregado resulta pertinente, principalmente, para las distintas colectividades nacionales que estarían alojando empleos de bajo valor agregado como consecuencia del proceso de deslocalización de las actividades de fabricación y ensamblado hacia los países en desarrollo. El caso de iPhone ilustra el proceso de marras: los buenos empleos -y la mayoría de los beneficios- están en California mientras que las actividades de ensamblaje de baja remuneración en China (Baldwin et. al., 2014).

Desde 1995, el traslado permanente de las actividades manufactureras desde las naciones desarrolladas hacia las economías en desarrollo resulta notable, especialmente a China. Efectivamente, mientras que esas actividades productivas representan un 3\% en 1990 en ese país asiático, luego de 20 años, en el 2010 alcanzan ya un 18\%. Correlativamente, entre las economías del $\mathrm{G} 7$, esas actividades representan $65 \%$ en 1990 y descienden en el 2010 a un 47\%. Ver Figura 1.

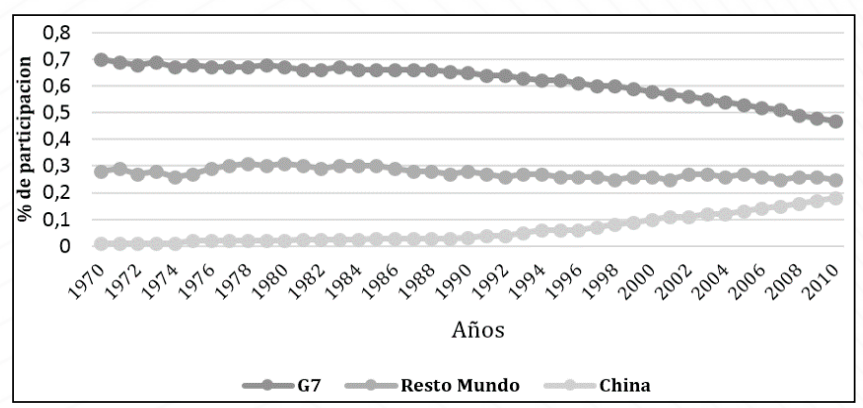

Fuente: Elaboración propia a partir del trabajo expuesto por Baldwin et. al. (2014) Figura 1. Desplazamiento de las actividades manufactureras mundiales

La deslocalización productiva parecería plasmarse en el desplazamiento del valor agregado desde la manufactura, su fase de fabricación, hacia los servicios pre y pos fabricación, la tercerización. A comienzos de 1990s, Stan Shih, fundador de Acer y CEO, populariza este itinerario como curva de la sonrisa. En el Estudio de Baldwin et. al., 2014, que desarrolla, en cambio, el concepto de curva agregada de la sonrisa intenta documentar, a través de una base de datos que incluye información de varios países asiáticos desde 1985, si la tercerización de la producción resulta un fenómeno mucho más reciente o si por el contrario ya se encuentra presente desde los 80's en esas economías. Para ese efecto se divide la información en dos periodos, 1985-1995, y, 1995-2005, estimando tanto las variaciones del valor agregado, $\Delta V A$, como el origen de los insumos utilizados en la cadena de valor distinguiendo entre fuentes primarias, secundarias y terciarias.

Los resultados de ese estudio se visualizan en la Figura No.2 según los cuales destaca una caída del valor agregado generado desde fuentes primarias entre 1985-1995, en Japón y Filipinas entre otras; en cambio, el valor originado en el sector manufacturero sube en ambos países en el mismo periodo, en tanto que la agregación de valor desde fuentes terciarias, se incrementa ligeramente en Japón y permanece sin modificación en Filipinas. Esa última evidencia, deja claro que en ese periodo no se constata el efecto tercerización en ninguna de esas economías. Los resultado esperados, sin embargo, se presentan con mayor nitidez en la década siguiente, 1995-2005. El valor añadido cae, conforme a lo esperado, desde las fuentes primarias en ambos países junto a una contracción del valor agregado generado desde fuentes secundarias. Se puede apreciar que la agregación de valor desde fuentes terciarias crece significativamente en Japón, en particular, junto a una caída del aporte que proviene de la fuente manufactura. En conjunto, el desplazamiento que sólo es visible sobre el lado izquierdo del gráfico sugiere, sin embargo, no precisamente una sonrisa sino una mueca -the smirk curve- particularmente para Filipinas (Baldwin et. al., 2014).

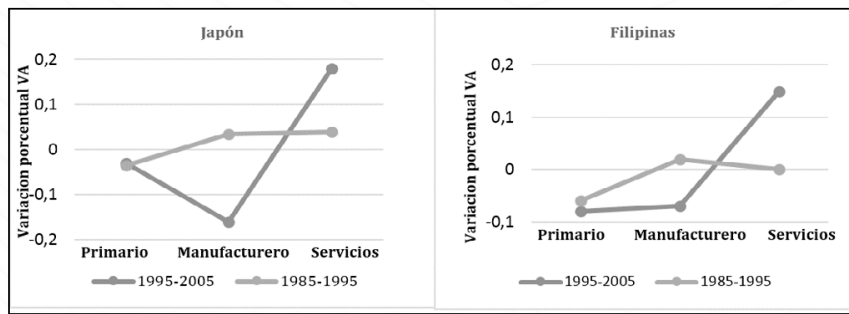

Fuente: Elaboración propia a partir del trabajo expuesto por Baldwin et. al. (2014) Figura 2. Curva agregada de la sonrisa de Japón y Filipinas según subperiodos

Sumariamente, estos resultados sugerirían que la fase manufactura en los países estudiados no se terceriza completamente y estaría más a tono con la recepción de empleos de ensamblaje de baja productividad que reducen el mark up en esas economías exceptuando Japón. Esta explicación, sin embargo, precisa de evidencia más concluyente dada la relevancia del sector servicios que vino acompañada por un desplazamiento de origen importante, desde el sector proveedores de servicios domésticos hacia los más dinámicos localizados en Japón y USA y en algunas de las propias economías emergentes.

Esta investigación, por su parte, estima descriptivamente los desplazamientos en las fuentes de agregación de valor utilizando el enfoque de la curva agregada de la sonrisa con el fin de determinar los efectos del Programa Semillas sobre una potencial tercerización de la producción entre los productores de la cadena de maíz en Ecuador. Para ese propósito este estudio capta las variaciones en los niveles de los insumos utilizados distinguiendo el origen de los mismos en base a información secundaria originada en las cuentas productivas y de costos de los diversos productores de la cadena. Mediante una aproximación de tipo corte-transversal, se estiman los desplazamientos entre las fuentes de agregación de valor a través de comparaciones entre las diversas estructuras productivas tomando como situación base la correspondiente al grupo productor tradicional no-intervenido donde predomina, en cambio, el uso de inputs de origen primario.

\section{Resultados}

El programa Semillas que transfiere paquetes tecnológicos combinando semillas certificadas de alto rendimiento, agroquímicos y fertilizantes de buena calidad intenta contribuir al mejoramiento de los rendimientos productivos frente a la opción de utilizar técnicas tradicionales de cultivo semillas recicladas, agroquímicos y fertilizantes de menor calidad- para enfrentar así la limitada producción interna y su dependencia de importaciones considerables de torta de soya y maíz (Inclusys, 2013).

El dinamismo de la cadena del maíz amarillo particularmente, de los segmentos asociados de exportación, como el de camarón y langostino, resultan cruciales -alrededor de USD 2 mil millones anuales-. Su aporte en valor al PIB total alcanza $2 \%$ promedio anual, mientras que su contribución al PIB agropecuario representa $21 \%$ anual en promedio. En su funcionamiento intervienen diversos agentes directos -productores de maíz; pequeños, medianos, y grandes acopiadores en el sector comercialización; firmas productoras de alimentos balanceados y de proteína animal entre otras-. La relación con la red de proveedores de 
insumos, de semillas y agroquímicos, es fundamental para los agentes del eslabón producción (Álvarez, 2016).

El insumo mano de obra es el principal costo entre los productores tradicionales que no cuentan con niveles de mecanización en ninguna etapa de la producción ni utilizan semilla certificada. Los costos indirectos que incluyen el valor de uso de la tierra, el financiamiento, administración y un monto destinado a imprevistos representan la mayor porción del costo. Le sigue en importancia el importe cosecha y post cosecha, junto a los costes de transporte, secado y limpieza que varían según el rendimiento. El productor tradicional no logra beneficios aceptables, por tanto, la transferencia tecnológica modifica la composición de los insumos productivos entre los productores beneficiarios.

Tabla 1. Costos de productores de maíz amarillo duro según actividad Año 2014.

\begin{tabular}{lcccccccc}
\hline & Tradicional & \multicolumn{3}{c}{$\begin{array}{c}\text { Tradicional } \\
\text { Beneficiario }\end{array}$} & $\begin{array}{c}\text { Semi } \\
\text { Tecnificado }\end{array}$ & \multicolumn{2}{c}{ Tecnificado } \\
\multicolumn{1}{c}{ Actividades } & Valor & $\%$ & Valor & $\%$ & Valor & $\%$ & Valor & $\%$ \\
\hline Preparación de suelo & 144 & $11 \%$ & 144 & $9 \%$ & 33 & $2 \%$ & 33 & $2 \%$ \\
Siembra & 200 & $15 \%$ & 340 & $21 \%$ & 498 & $27 \%$ & 580 & $29 \%$ \\
Fase Vegetativa & 307 & $22 \%$ & 398 & $24 \%$ & 470 & $26 \%$ & 384 & $19 \%$ \\
Cosechay Pos cosecha & 340 & $25 \%$ & 363 & $22 \%$ & 398 & $22 \%$ & 528 & $27 \%$ \\
Indirectos & 380,3 & $28 \%$ & 380,3 & $23 \%$ & 433,8 & $24 \%$ & 450,5 & $23 \%$ \\
Total & $1.371,30$ & $100 \%$ & $1.625,30$ & $100 \%$ & $1.832,80$ & $100 \%$ & $1.975,50$ & $100 \%$ \\
\hline
\end{tabular}

Fuente: Elaboración propia a partir del trabajo expuesto por Álvarez. (2016)

Efectivamente esa influencia se puede apreciar en los resultados de las cuentas productivas del productor tradicional y tradicional-beneficiario. Entre los cambios por actividad que destaca refiere a la actividad siembra, cuyo valor pasa de 200 dólares a USD 340 por hectárea junto a la actividad fase vegetativa que llega a 398 por arriba de los USD 307 dólares por hectárea entre los productores no intervenidos.

Los productores semi-tecnificados, por su lado, preparan el terreno con maquinaria, realizan la cosecha y desgranada de forma mecánica. La actividad de siembra ocupa la mayor participación en su estructura productiva, 27\%; cosecha y post cosecha, representa $22 \%$. Estos productores alcanzan por tanto altos niveles de productividad. Por su parte, el costo del productor tecnificado refleja su alto grado de mecanización representando 34\% de participación; así mismo destacan los costos en semillas y fertilizantes.

Esa composición que enfatiza en fuentes secundarias permite incrementar los rendimientos del cultivo considerablemente; el uso de la tierra representa todavía un costo importante entre todos los productores. Los costos totales de producción por hectárea se presentan detalladamente en la Tabla 2 a partir de los cuales emerge la estructura tecnológica subyacente a los distintos productores.

Tabla 2. Costos de producción de maíz por hectárea por rubro según tipo de productor. Año 2014.

\begin{tabular}{|c|c|c|c|c|}
\hline $\begin{array}{l}\text { Costo hectárea } \\
\text { Pago de factores }\end{array}$ & Tradicional & $\begin{array}{l}\text { Tradicional } \\
\text { Beneficiario }\end{array}$ & $\begin{array}{l}\text { Semi } \\
\text { Tecnificado }\end{array}$ & Tecnificado \\
\hline Mano de obra & 576 & 384 & 264 & 120 \\
\hline Uso tierra & 250 & 250 & 250 & 250 \\
\hline Maquinaria/equipo & 148,5 & 267,2 & 500 & 676 \\
\hline Costos indirectos & 79,3 & 175,2 & 111,8 & 122 \\
\hline Costos financiamiento & 51 & 51 & 72 & 78,5 \\
\hline Semilla & 80 & 220 & 220 & 240 \\
\hline Fertilizante & 143,4 & 221,6 & 313,6 & 336,8 \\
\hline Agroquímicos & 43,1 & 56,2 & 100,5 & 122,1 \\
\hline Total & $1.371,3$ & $1.625,2$ & $1.831,9$ & 1975,4 \\
\hline Subsidio & & -214 & & \\
\hline Total USD/hec & $1.371,3$ & $1.411,2$ & $1.831,9$ & $1.975,4$ \\
\hline Rendimiento seco $q q / h e c$ & 81 & 114 & 128 & 156 \\
\hline Costo unitario USD/qq S/S & 16,9 & 14,38 & 14,31 & 12,66 \\
\hline Costo unitario USD/qq C/S & & 12,49 & & \\
\hline
\end{tabular}

Los productores tradicionales por su bajo nivel de tecnificación, uso de material genético reciclado, mantienen rendimientos bajos y generan costos de producción unitarios altos. Su costo unitario, 16,9 USD/qq, ni cubre el valor del precio mínimo de sustentación del 2014, 15,90 USD/qq. Del costo total, mano de obra representa $42 \%$, el uso de tierra ocupa una porción importante, 18\%. Los bajos rendimientos impiden apalancar costos fijos como el uso de tierra, utilización de semillas y fertilizantes de calidad. Los beneficiarios por la transferencia tecnológica presentan un cambio importante; el costo promedio resulta muy similar al costo de los más tecnificados no-beneficiarios y sus rendimientos superan en $40 \%$ al generado en el cultivo tradicional. El costo unitario cae a 12,5 USD/qq, 26\% menos, e incluye un cambio en la estructura de costos, la mano de obra pasa de $42 \%$ a $24 \%$ a favor de incrementos en el valor de semilla, fertilizante y maquinaria y equipo.

Entre los productores semi-tecnificados los costos de semilla, fertilizantes y mecanización son muy superiores respecto a los del productor tradicional lo que mejora el rendimiento con base a un costo unitario más bajo; al precio mínimo de sustentación, 15,90, la utilidad generada llega a $11 \%$ aproximadamente. La estructura del productor tecnificado, por su parte, evidencia incrementos en los costos de semilla, fertilizantes y mecanización respecto del productor tradicional, esto incide en rendimientos más altos y en un costo unitario más bajo; al precio mínimo de sustentación la utilidad representa 26\% aproximadamente.

A partir las cuentas productivas de los productores se estiman los desplazamientos en las fuentes de agregación de valor en términos de variaciones en los niveles de los insumos utilizados; la aproximación es de corte-transversal ya que la información disponible consta de datos sólo para el 2014. Por falta de data específica sobre el insumo terciario, se utiliza, el componente beneficios del valor agregado como su valor proxi, es decir, el mark up, generado estrictamente entre los productores que utilizan insumos de alto rendimientos. La identificación de las fuentes de agregación de valor entre los diferentes productores distingue, según el enfoque metodológico de la curva agregada, fuentes primarias y secundarias. Ver Tabla 3.

Tabla 3. Agregación de valor según fuentes e insumos, cifras en miles USD

\begin{tabular}{lrrrrr}
\hline \multicolumn{1}{c}{ Productores } & $\begin{array}{c}\text { Valor } \\
\text { Mercado }\end{array}$ & Primario & $\begin{array}{c}\text { Valor Agregado } \\
\text { Secundario }\end{array}$ & Mark up & $\begin{array}{c}\text { Insumos } \\
\text { Intermedios }\end{array}$ \\
\hline $\begin{array}{l}\text { Tradicional No } \\
\text { Beneficiario }\end{array}$ & 26.301 & 18.114 & 5.320 & -2.388 & 5.255 \\
$\begin{array}{l}\text { Tradicional } \\
\text { Beneficiario }\end{array}$ & 86.373 & 34.969 & 20.651 & -3.335 & 34.088 \\
$\begin{array}{l}\text { Productos Semi } \\
\text { Tecnificado }\end{array}$ & 301.860 & 79.086 & 103.474 & 26.166 & 93.135 \\
$\begin{array}{l}\text { Productor } \\
\text { Tecnificado }\end{array}$ & 39.603 & 6.076 & 14.396 & 7.601 & 11.529 \\
\hline & Fuente: Elaboración propia a partir del trabajo expuesto por Álvarez. (2016)
\end{tabular}

Los desplazamientos estimados entre los diversos agentes productores -tradicionales, semi-tecnificados y tecnificados- excluyen los insumos intermedios y compara los cambios registrados en los niveles y utilización de los mismos en un solo periodo tomando como situación-base la estructura del grupo tradicional no-intervenido cuya operación se centra en el uso de fuentes primarias.

Tabla 4. Desplazamiento del valor agregado desde las fuentes e insumos

\begin{tabular}{lccc}
\hline \multicolumn{1}{c}{ Productores } & Primario & Secundario & Terciario \\
\hline Tradicional Beneficiario & $-0,1918611$ & 0,1421781 & 0,0496830 \\
& & & \\
Productos Semi Tecnificado & $-0,4817867$ & 0,2429519 & 0,2388348 \\
Productor Tecnificado & $-0,644243$ & 0,2600079 & 0,3842351
\end{tabular}

Fuente: Elaboración propia a partir del trabajo expuesto por Álvarez. (2016) 
Las estimaciones evidencian una caída importante en la participación de los insumos primarios y un aumento simultáneo de la influencia de los inputs manufactureros para todos productores no tradicionales, junto a importantes desplazamientos hacia los insumos terciarios, particularmente entre los productores tecnificados y semi-tecnificados que evidentemente generan mayores márgenes y mark up. Interesa, sin embargo, destacar la situación de los productores tradicionales, el beneficiario del Programa y el no-intervenido.

Como se puede advertir en la Figura 3, la situación que emerge para el productor beneficiario frente al tradicional implica una importante sustitución de las fuentes de agregación primarias hacía, principalmente, las secundarias. Este desempeño no resulta, empero, tan satisfactorio según lo que predice la curva de la sonrisa que por el contrario busca el afianzamiento del insumo terciario como resultado de una pérdida progresiva en la importancia del insumo secundario. Los resultados expuestos en la Tabla 4 evidenciarían que la situación de los productores beneficiarios no resulta muy diferente para los productores tecnificados y semitecnificados.

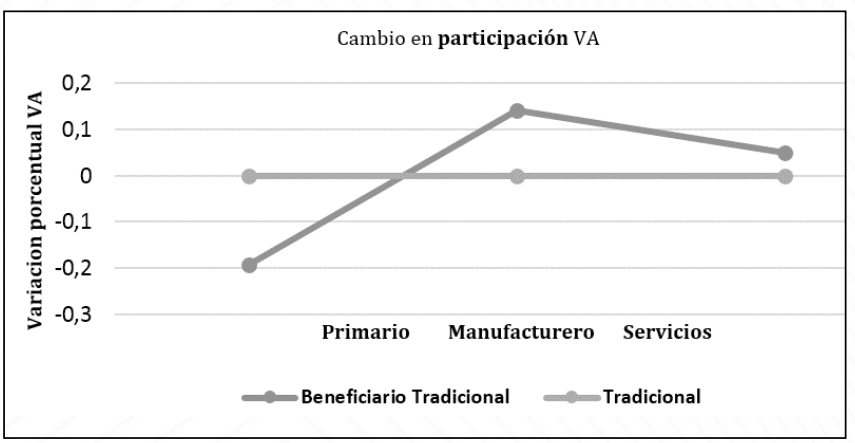

Fuente: Elaboración propia a partir del trabajo expuesto por Álvarez. (2016) Figura 3. Cambio en la agregacion de valor del productor tradicional beneficiario

\section{Conclusiones}

Las estimaciones y resultados sobre el desplazamiento en las fuentes de agregación de valor entre los diversos insumos -primarios, secundarios y terciarios- y a través de los distintos tipos de productores que convergen en la cadena de maíz de Ecuador, sumariamente, sugerirían que los cambios detectados no siguen la inclinación que el estudio de Baldwin et. al., encuentra en varios países asiáticos, incluido Japón para el periodo 1995-2005 en donde se visualiza con mucha claridad que el proceso de agregación de valor emergente sigue una forma aproximada a la curva agregada de la sonrisa y por tanto se infiere una progresiva tercerización, aunque incompleta, de la producción en esas economías y en lapso temporal. Ver Figura No. 2

Los desplazamientos en las fuentes de agregación de valor encontrados en la cadena de maíz amarillo ecuatoriana por este estudio, en cambio, siguen la dirección que establece Baldwin et. al., 2014, en esos mismos países y en todos sus sectores económicos en el periodo previo, es decir, entre 1985-1990. Aquí, sin embargo, la situación encontrada no precisamente evidenciaría un proceso compatible de tercerización de la producción, dado el predominio de las fuentes secundarias de agregación de valor. El análisis más completo de ambos periodos en la experiencia asiática, muestra que esta fase, empero, constituye una fase precursora que allana el sendero hacia la tercerización. Aunque los resultados detectados en la cadena de maíz ecuatoriana resultan similares a los de la primera fase asiática y estaría presente entre todos sus productores, incluidos los tradicionales-beneficiarios, y particularmente, entre los tecnificados y semi-tecnificados que reemplazan y sustituyen insumos primarios, no permiten concluir, sin embargo, que la experiencia de los productores de la cadena de maíz constituye una verdadera antesala hacia la tercerización.

Sumariamente, la experiencia asiática transita entre 1985-2005 desde la fase en que predominan las fuentes secundarias hacia la tercerización de la producción, especialmente, según la evidencia de Baldwin, en
Japón y con menor claridad en el resto de economías como el caso de Filipinas. Los cambios detectados en la cadena de maíz ecuatoriana se localizan en la fase caracterizada por un predominio mucho más arraigado y dependiente de los insumos de origen secundario, permaneciendo todavía anclada al ámbito de baja agregación de valor y productividad. A la luz de este análisis emerge, consiguientemente, la importancia y los desafíos en torno a la necesidad de sostener políticas competitivas estructuradas alrededor del insumo terciario que resultan cruciales para catalizar y materializar el tránsito hacia fases más avanzadas superando aquellas en que gravitan los input primarios y secundarios.

Estos hallazgos requieren evidentemente de estimaciones más comprehensivas sustentadas en variaciones a lo largo del tiempo de las fuentes de agregación de valor, incluyendo una base de información mucho más abarcativa sobre el comportamiento de otras cadenas y sectores productivos para lograr determinaciones más concluyentes que faculten confirmar que la situación aquí detectada corresponda en realidad, a la fase previa de tercerización de la producción conforme a la experiencia asiática según la investigación de Baldwin et al, 2014.

\section{Referencias}

Acemuglu D. y J. Robinson (2012). Por que fracasan los paises. Colombia: Deusto

Alvarez Pablo. (2016). Análisis económico de la politica implementada en la cadena productiva del maiz amarillo duro en Ecuador, periodo 2010-2015. Quito: Mimeo

Banco Mundial. (2008). Agricultura para el desarrollo. Washington, D.C.: Banco Mundial.

Baldwin R., R Forslid and T., Ito (2015). Unveiling the evolving sources of value added in exports.

www.ide.go.jp/library/English/Publish/Download/Jrp/.../161.pdf

Baldwin R., R Forslid and T., Ito (2014). The smile cruve: Evolving sources of value added in manufacturing http//www.rieti.go.jp/en/events/14121101/ pdf/baldwin.pdf

Bellù, L. (2013). Value Chain analysis for Policy Making. Methodological Guidelines and country cases for a Quantitative Approach. Roma: Food and Agriculture Organization.

BCE (2014). Reporte de coyuntura sector agropecuario. Quito: Banco Central del Ecuador.

CEPAL (2008). Análisis de los mercados de materias primas agrícolas y de los precios de los alimentos. Santiago de Chile: Unidad de Desarrllo Agrícola, Comisión Económica para América Latina y el Caribe.

IICA (s.f.). Sistema agroproductivo, cadenas y competitividad. Lima: Instituto Interamericano de Cooperación para la Agricultura.

Inclusys. (2013). Plan de Mejora Competitivo de la Cadena Balanceados. Diagnóstico de la Cadena de Balanceados. Quito.

INEC (2000). Censo Nacional Agropecuario. Quito: INEC

Helpman Elhanan (2007) El Misterio del Crecimiento Economico.

España: Antoni Bosch

MAGAP (2013). Proyecto Nacional de Semillas para Agrocadenas Estratégicas. Quito.

Moreno, B., \& Salvador, S. (2015). Determinantes del rendimiento del cultivo de maíz duro seco. Quito: Coordinación General del Sistema de Información Nacional.

SENPLADES. (2014). Proyecto nacional para el desarrollo integral de cadenas agroindustriales. Quito. 